\title{
VARIATIONAL METHODS FOR FUNCTIONS WITH POSITIVE REAL PART
}

BY

\section{S. ROBERTSON}

1. Introduction. M. M. Schiffer [8] has recently derived a formula for the variation of the Green's function of the most general plane domain D with boundary $\mathfrak{C}$ due to a small shift

$$
w^{*}=w+\rho^{2} \phi(w),
$$

of the boundary. The variation $\delta g(\zeta, \eta)$ of the Green's function is given by the formula

$$
\delta g(\zeta, \eta)=\operatorname{Re}\left\{\frac{\rho^{2}}{2 \pi i} \oint_{\Gamma} p^{\prime}(w, \eta) p^{\prime}(w, \zeta) \phi(w) d w\right\}+o\left(\rho^{2}\right)
$$

where $p(w, \eta)$ is an analytic function whose real part is the Green function $g(w, \eta)$ of $D$, and where $\Gamma$ is a member of a curve system in $D$ homotopic to e. The function $\phi(w)$ is analytic on $\Gamma$ and in the ring bounded by $\mathfrak{C}$ and $\Gamma$. If $D$ is simply-connected and if $z=\psi(w)$ maps $D$ on the interior of the unit circle $|z|<1$, then $g(w, \eta)$ is connected with $\psi(w)$ by the relation

$$
g(w, \eta)=\log \left|\frac{1-(\psi(\eta))-\psi(w)}{\psi(w)-\psi(\eta)}\right| .
$$

Here and throughout the paper ( )- indicates the complex conjugate. With an appropriate choice of $\phi(w)$ one may then obtain variation formulas for univalent functions $w=f(z)$. J. A. Hummel [5] has recently used this method of interior variations to study the class of univalently star-like functions. The method may also be used to study those functions which are convex-inone direction [2]. The choice of the shift function $\phi(w)$, however, is not always an obvious one for many special classes of univalent functions, in particular for the class of close-to-convex functions [6]. Many of these special classes, however, have representations of their member functions in terms of functions $P(z)$ with positive real part. It therefore becomes desirable to have a variational formula for $P(z)$ from which one may then easily obtain analogous variational formulas for the special classes of univalent functions.

It is the purpose of this paper first to derive a variational formula for the class $P$ of normalized regular functions

$$
P(z)=1+p_{1} z+p_{2} z^{2}+\cdots+p_{n} z^{n}+\cdots, \quad P(0)=1,
$$

Received by the editors April 7, 1961. 
which have $\operatorname{Re} P(z)>0$ in $|z|<1$. Secondly, we shall apply the variational formula to $P(z)$ in order to solve extremal problems for the class $\beta$ and in particular to obtain a characterization of the $(n-1)$ Euclidean coefficient space $E_{n-1}$ for the extremal functions $P(z)$ for which $\operatorname{Re} p_{n}$ is a maximum. Although these results may also be deduced from the Carathéodory-Toeplitz theory $[1 ; 3 ; 4 ; 7 ; 9 ; 10]$ it is interesting to see how simply they are derived by the variational method. Thirdly, we shall indicate how the variational formula for $P(z)$ leads to the variational formulas for bounded, regular functions $\omega(z)$ and also for regular functions $F(z)=f\{\omega(z)\}$ which are subordinate to a given univalent function $f(z)$ in $|z|<1$.

2. A variational formula for the class $P$. The choice

$$
\phi(w)=\frac{e^{i \theta}}{w-w_{0}}
$$

in (1.2), where $w_{0}$ is interior to $D$, is the Schiffer case for univalent functions [8]. Hummel [5] chose

$$
\begin{array}{rlrl}
\phi(w) & =w R[\psi(w)], \\
R(z) & =e^{i \theta} \cdot \frac{1-\bar{z}_{0} z}{z-z_{0}}+e^{-i \theta} \cdot \frac{z-z_{0}}{1-\bar{z}_{0}}, & & \left|z_{0}\right|<1,
\end{array}
$$

where $R(z)$ is real and bounded on $|z|=1$, to obtain the following variational formula for a normalized univalently star-like function $f(z)$ in $|z|<1, f(0)=0$, $f^{\prime}(0)=1$ :

$$
f^{*}(z)=f(z)\left[1-\rho^{2}\left(1-\left|z_{0}\right|^{2}\right)\left(A(z)-\frac{z f^{\prime}(z)}{f(z)} B(z)\right)\right]+o\left(\rho^{2}\right)
$$

where the error term $o\left(\rho^{2}\right)$ is an analytic function in $z$ and uniformly bounded in each interior region of $|z|<1 . A(z)$ and $B(z)$ are defined by

$$
\begin{array}{ll}
A(z)=\frac{z e^{i \theta}}{z_{0}\left(z-z_{0}\right)}+\frac{z e^{-i \theta}}{1-\bar{z}_{0} z}-\frac{e^{i \theta} f\left(z_{0}\right)}{z_{0}^{2} f^{\prime}\left(z_{0}\right)}, & \left|z_{0}\right|<1, \\
B(z)=\frac{e^{i \theta} f\left(z_{0}\right)}{z_{0} f^{\prime}\left(z_{0}\right)\left(z-z_{0}\right)}-e^{-i \theta}\left(\frac{f\left(z_{0}\right)}{z_{0} f^{\prime}\left(z_{0}\right)}\right)^{-} \frac{z}{1-\bar{z}_{0} z} .
\end{array}
$$

Since there is a (1-1) correspondence between the functions $P(z)$ of the class $P$, given by (1.4), and the univalently star-like functions $f(z), f(0)=0$, $f^{\prime}(0)=1$, by the relation

$$
P(z)=\frac{z f^{\prime}(z)}{f(z)}, \quad \operatorname{Re} P(z)>0, \quad|z|<1,
$$

we therefore take the logarithmic derivative in (2.2) and replace $z\left(f^{* \prime}(z)\right) / f^{*}(z)$ by $P^{*}(z)$. After some simplification we obtain 


$$
\begin{aligned}
& P^{*}(z)=P(z)-\rho^{2}\left(1-\left|z_{0}\right|^{2}\right) z \\
& \cdot\left[\frac{z e^{i \theta}}{z_{0}\left(z-z_{0}\right)}+\frac{z e^{-i \theta}}{1-\bar{z}_{0} z}-\frac{e^{i \theta} P(z)}{P\left(z_{0}\right)\left(z-z_{0}\right)}+\frac{e^{-i \theta} z P(z)}{\left(P\left(z_{0}\right)\right)^{-}\left(1-\bar{z}_{0} z\right)}\right]^{\prime}+o\left(\rho^{2}\right),
\end{aligned}
$$

where [ ]' denotes differentiation with respect to $z$. Let $\delta P(z)=P^{*}(z)-P(z)$, and write (2.6) in the form

$$
\begin{array}{r}
\frac{-\delta P(z)}{\rho^{2}\left(1-\left|z_{0}\right|^{2}\right)}=\left(\frac{z_{0} P^{\prime}(z)}{P\left(z_{0}\right)}-1\right) \frac{e^{i \theta} z}{z_{0}\left(z_{0}-z\right)}+\left(\frac{z_{0} P(z)}{P\left(z_{0}\right)}-z\right) \frac{e^{i \theta} z^{4}}{z_{0}\left(z_{0}-z\right)^{2}} \\
+\frac{P^{\prime}(z)}{\left(P\left(z_{0}\right)\right)^{-}}\left(\frac{z^{2} e^{-i \theta}}{1-\bar{z}_{0} z}\right)+\left(\frac{P(z)}{\left(P\left(z_{0}\right)\right)^{-}}+1\right) \frac{e^{-i \theta} z}{\left(1-\bar{z}_{0} z\right)^{2}}+o(1),
\end{array}
$$

which is the required variational formula for functions of class $P$.

If $P(z)$ has the power series expansion (1.4), and if we denote $\delta p_{n}=p_{n}^{*}-p_{n}$, (2.7) yields

$$
\begin{aligned}
\frac{-\delta p_{n}}{\rho^{2}\left(1-\left|z_{0}\right|^{2}\right)}= & \frac{n e^{i \theta}}{P\left(z_{0}\right)} \sum_{k=0}^{n} \frac{p_{k}}{z_{0}^{n-k+1}}-\frac{n e^{i \theta}}{z_{0}^{n+1}}+\frac{n e^{-i \theta}}{\left(P\left(z_{0}\right)\right)^{-}} \sum_{k=0}^{n-1} p_{k}\left(\bar{z}_{0}\right)^{n-k-1}, \\
& +n e^{-i \theta}\left(\bar{z}_{0}\right)^{n-1}+o(1)
\end{aligned}
$$

where $p_{0}=1$. Since for any complex number $w, \operatorname{Re} w=\operatorname{Re} \bar{w}$, we have

$$
\begin{aligned}
\frac{-\operatorname{Re} \delta p_{n}}{\rho^{2}\left(1-\left|z_{0}\right|^{2}\right)}=n \operatorname{Re}\left\{\frac { e ^ { i \theta } } { z _ { 0 } } \left[\frac{1}{P\left(z_{0}\right)} \sum_{k=0}^{n-1}\left(\frac{p_{k}}{z_{0}^{n-k}}+p_{k} z_{0}^{n-k}\right)\right.\right. \\
\left.\left.+\frac{p_{n}}{P\left(z_{0}\right)}+z_{0}^{n}-z_{0}^{-n}\right]\right\}+o(1) .
\end{aligned}
$$

3. Extremal functions for the class $\odot$. For any positive integer $n$ let $P(z)$ be an extremal function of the class $P$ for which the coefficient $p_{n}$ in (1.4) is real, positive and a maximum over all functions of the class. Since $P$ is compact $p_{n}$ attains its maximum. Its value is 2 as is well known from the Carathéodory theory, although we do not assume this fact here. Since $\operatorname{Re} \delta p_{n} \leqq 0$ in (2.9) and $\theta$ is arbitrary we have from (2.9) on replacing $z_{0}$ by $z$

$$
\begin{aligned}
& \frac{1}{P(z)}\left\{p_{n}+\sum_{k=0}^{n-1}\left(\frac{p_{k}}{z^{n-k}}+p_{k} z^{n-k}\right)\right\}+z^{n}-z^{-n}=0, \quad p_{n}>0, p_{0}=1 \\
& P(z)=\frac{1+p_{1} z+p_{2} z^{2}+\cdots+p_{n} z^{n}+\cdots+p_{2 n-1} z^{2 n-1}+z^{2 n}}{1-z^{2 n}}=\frac{Q_{2 n}(z)}{1-z^{2 n}}
\end{aligned}
$$

where $p_{2 n-k}=p_{k}, k=1,2, \cdots, 2 n-1$.

Although ordinarily the variational formulas for extremal functions of various classes of functions lead to differential equations we find here for the 
class $\beta$ that we are led directly to the extremal functions (3.2) without encounting a differential equation. However, the formula (3.2) may be simplified further. We shall presently show that whenever $P(z)$ has the form (3.2) and has a positive real part in $|z|<1$ then $p_{n} \leqq 2$, and if $p_{n}=2$ then $\left(1+z^{n}\right)$ is a factor of both numerator and denominator of (3.2). In this case (3.2) becomes

$$
P(z)=\frac{1+p_{1} z+p_{2} z^{2}+\cdots+p_{n-1} z^{n-1}+z^{n}}{1-z^{n}},
$$

where $p_{n-k}=p_{k}, k=1,2, \cdots, n-1$.

To obtain (3.3) we place $z=r e^{k \pi i / n}, 0<r<1, k=$ odd integer, in (3.2). Then

$$
P\left(r e^{k \pi i / n}\right)=\frac{Q_{2 n}\left(r e^{k \pi i / n}\right)}{1-r^{2 n}}
$$

Since $\operatorname{Re} P(z)>0$ it follows that $\operatorname{Re} Q_{2 n}\left(r e^{k \pi i / n}\right)>0$. Letting $r \rightarrow 1$ we have $\operatorname{Re} Q_{2 n}\left(e^{k \pi i / n}\right) \geqq 0$. However, $Q_{2 n}\left(e^{k \pi i / n}\right)$ is real. This follows since $p_{\nu} z^{\nu}+p_{2 n-v} z^{2 n-\nu}$ $=p_{\nu} z^{\nu}+p_{v} z^{-v}=$ a real number when $z=e^{k \pi i / n}$, and because $p_{n} z^{n}$ and $z^{2 n}$ are also real for this choice of $z$. Thus

$$
Q_{2 n}\left(e^{k \pi i / n}\right) \geqq 0 .
$$

Let

$$
\begin{aligned}
& P_{n-1}(z)=\sum_{s=1}^{n-1} p_{s} z^{2} \\
& Q_{2 n}(z)=1+p_{n} z^{n}+z^{2 n}+P_{n-1}(z)+z^{2 n} \cdot\left(P_{n-1}\left(\frac{1}{\bar{z}}\right)\right)^{-} .
\end{aligned}
$$

For $k$ odd, $z=e^{k \pi i / n}$, we have $z^{2 n}=1, z^{n}=-1$, so that

$$
\begin{aligned}
& 0 \leqq Q_{2 n}\left(e^{k \pi i / n}\right)=\left(2-p_{n}\right)+P_{n-1}\left(e^{k \pi i / n}\right)+\left(P_{n-1}\left(e^{k \pi i / n}\right)\right)- \\
& 0 \leqq\left(2-p_{n}\right)+2 \operatorname{Re} P_{n-1}\left(e^{k \pi i / n}\right)=\left(2-p_{n}\right)+2 \operatorname{Re}\left(\sum_{e=1}^{n-1} p_{e} e^{k \varepsilon \pi i / n}\right) .
\end{aligned}
$$

By virtue of the identity

$$
\sum_{v=1}^{n} e^{(2 v-1) e \pi i / n}=0, \quad s=1,2, \cdots, n-1
$$

it follows that

$$
\operatorname{Re} \sum_{n=1}^{n} P_{n-1}\left(e^{(2 v-1) \pi i / n}\right)=0
$$

From (3.8) and (3.10) we then have 


$$
0 \leqq \sum_{\nu=1}^{n}\left[2-p_{n}+2 \operatorname{Re} P_{n-1}\left(e^{(2 \nu-1) \pi i / n}\right)\right]=n\left(2-p_{n}\right)
$$

Thus $p_{n} \leqq 2$. But since $\left(1+2 \sum_{1}^{\infty} z^{n}\right)$ is a member of class $\beta$ and $p_{n}$ is maximal we must have $p_{n}=2$. In this case (3.8) reduces to

$$
\operatorname{Re} P_{n-1}\left(e^{k \pi i / n}\right) \geqq 0,
$$

$k$ odd.

However, only equality can hold in (3.12) since otherwise (3.10) would be contradicted. (3.7) now becomes

$$
Q_{2 n}\left(e^{k \pi i / n}\right)=2 \operatorname{Re} P_{n-1}\left(e^{k \pi i / n}\right)=0,
$$

$k$ odd.

It follows at once that $\left(1+z^{n}\right)$ is a factor of $Q_{2 n}(z)$.

If we set

$$
1+z^{2 n}+\sum_{s=1}^{2 n-1} p_{s} z^{s}=Q_{2 n}(z)=\left(1+z^{n}\right) \sum_{0}^{n} q_{s} z^{8}
$$

where $p_{2 n-s}=p_{8}, s=1, \cdots, 2 n-1$, and $p_{n}=2$, we find that

$$
\begin{array}{ll}
q_{s}=p_{s}, & s=1,2, \cdots, n-1 ; \\
q_{0}=q_{n}=1 ; & \\
p_{s}=p_{2 n-s}=q_{n-s}=p_{n-s}, & s=1,2, \cdots, n-1 .
\end{array}
$$

Thus we have shown that (3.3) follows from (3.2).

It is interesting to observe also that the extremal functions of (3.3) satisfy the identity

$$
P(z)+\left(P\left(\frac{1}{\bar{z}}\right)\right)^{-}=0 .
$$

Consequently, the real part of $P(z)$ vanishes identically on $|z|=1$.

If we let $\omega_{k}=e^{2 k \pi i / n}, k=1,2, \cdots, n$, we may write $P(z)$ of $(3.3)$ in the form

$$
P(z)=\sum_{k=1}^{n} \lambda_{k}\left(\frac{1+\omega_{k} z}{1-\omega_{k} z}\right), \quad 0 \leqq \lambda_{k} \leqq 1, \sum_{1}^{i} \lambda_{k}=1
$$

$\operatorname{Re} P(z) \equiv 0$ on $|z|=1$. But if we let $z=e^{i \theta} \rightarrow \bar{\omega}_{\nu}$ we find that the real part of the right-hand side of equation (3.16) is unbounded unless $\lambda_{\nu}$ is real. Moreover $\lambda_{\nu} \geqq 0$. For if we assume $\lambda_{\nu} \neq 0$ and let $z=r \bar{\omega}_{\nu}, 0<r<1$, as $r$ approaches 1 we find that $\operatorname{Re} P(z)$ must coincide in sign with that of $\lambda_{\nu}$. Furthermore

$$
\sum_{1}^{n} \lambda_{k}=P(0)=1 \text {. }
$$

It follows from (3.3) and (3.16) that the coefficients $p_{n}$ in (3.3) must be expressible in terms of the barycentric coordinates $\lambda_{k}$ as follows: 


$$
\left\{\begin{array}{rlrl}
p_{\nu} & =2 \sum_{k=1}^{n} \lambda_{k} e^{2 \nu k \pi i / n}, & 0 \leqq \lambda_{k} \leqq 1, \sum_{1}^{n} \lambda_{k}=1, & \nu=1,2, \cdots, n ; \\
p_{n-\nu} & =p_{p}, & \nu \leqq n-\nu, 1 \leqq \nu<n .
\end{array}\right.
$$

Conversely, if $0 \leqq \lambda_{k} \leqq 1, \sum_{1}^{n} \lambda_{k}=1$, then $P(z)$ given by (3.16) has $\operatorname{Re} P(z)>0$, $|z|<1$.

Let $p_{k}=x_{k}+i y_{k}$. Since $p_{n-k}=p_{k}$, it is seen that the coefficient space $E_{n-1}$ of the extremal functions $P(z)$, for which $p_{n}=2$, depends upon $(n-1)$ real variables $x_{k}, y_{k}$.

$$
\begin{aligned}
E_{n-1} & =E_{n-1}\left(p_{1}, p_{2}, \cdots, p_{(n-1) / 2}\right) \\
& =E_{n-1}\left(x_{1}, y_{1}, x_{2}, y_{2}, \cdots, x_{(n-1) / 2}, y_{(n-1) / 2}\right)
\end{aligned}
$$

for $n$ odd $>1$, and

$$
\begin{aligned}
E_{n-1} & =E_{n-1}\left(p_{1}, p_{2}, \cdots, p_{n / 2}\right) \\
& =E_{n-1}\left(x_{1}, y_{1}, \cdots, x_{(n-2) / 2}, y_{(n-2) / 2}, x_{n / 2}\right)
\end{aligned}
$$

for $n$ even. We define $E_{0}$ to be the point $p_{1}=2$ corresponding to the extremal $P(z)=(1+z) /(1-z)$.

From (3.17) it is readily seen that $E_{1}\left(p_{1}\right)$ is the closed 1 -simplex, or line segment $-2 \leqq p_{1} \leqq 2$, corresponding to

$$
P(z)=\frac{1+p_{1} z+z^{2}}{1-z^{2}}, \quad p_{1} \text { real, }-2 \leqq p_{1} \leqq 2 .
$$

$E_{2}\left(p_{1}\right)$ is a closed 2-simplex consisting of an equilateral triangle with vertices $(2,0),\left(-1,3^{1 / 2}\right)$ and $\left(-1,-3^{1 / 2}\right)$ and $E_{2}$ corresponds to the extremal functions of the form

$$
P(z)=\frac{1+p_{1} z+p_{1} z^{2}+z^{3}}{1-z^{3}}, \quad p_{1} \subset E_{2} .
$$

$E_{3}\left(p_{1}, p_{2}\right)$ is a tetrahedron with vertices $(0,2,-2),(0,-2,-2),(-2,0,2)$ and $(2,0,2) . E_{3}$ corresponds to the extremal function

$$
P(z)=\frac{1+p_{1} z+p_{2} z^{2}+p_{1} z^{3}+z^{4}}{1-z^{4}},
$$

where $p_{2}$ is real.

In general $E_{n-1}$ is the closed (n-1)-simplex with the $n$ vertices:

$$
\begin{aligned}
\left(2 \cos \frac{2 \nu \pi}{n}, 2 \sin \frac{2 \nu \pi}{n}, 2 \cos \frac{4 \nu \pi}{n}, 2 \sin \frac{4 \nu \pi}{n}, \ldots,\right. \\
\left.2 \cos (n-2) \frac{\nu \pi}{n}, 2 \sin (n-2) \frac{\nu \pi}{n}, 2 \cos \nu \pi\right),
\end{aligned}
$$


$\nu=1,2, \cdots, n$, when $n$ is even, or

$$
\begin{aligned}
\left(2 \cos \frac{2 \nu \pi}{n}, 2 \sin \frac{2 \nu \pi}{n}, 2 \cos \frac{4 \nu \pi}{n},\right. & 2 \sin \frac{4 \nu \pi}{n}, \ldots, \\
& \left.2 \cos (n-1) \frac{\nu \pi}{n}, 2 \sin (n-1) \frac{\nu \pi}{n}\right)
\end{aligned}
$$

if $n$ is odd.

The boundary hyperplanes of $E_{n-1}$ (corresponding to a $\lambda_{k}=0$ ) for $n$ odd, $n>1$, have the equations, for $k=0,1, \cdots, n-1$,

$$
1+\sum_{m=1}^{(n-1) / 2}\left(x_{m} \cos \frac{2 k m \pi}{n}-y_{m} \sin \frac{2 k m \pi}{n}\right)=0 ;
$$

for $n$ even, $n>2$, the equations of the hyperplanes are

$$
1+\frac{(-1)^{k}}{2} x_{n / 2}+\sum_{m=1}^{(n-2) / 2}\left(x_{m} \cos \frac{2 k m \pi}{n}-y_{m} \sin \frac{2 k m \pi}{n}\right)=0
$$

where $p_{m}=x_{m}+i y_{m}$.

It is seen that the hyperplanes (3.25) and (3.26) are tangent to the spheres

$$
\sum_{m=1}^{(n-1) / 2}\left(x_{m}^{2}+y_{m}^{2}\right)=\frac{4}{2 n-2}, \quad x_{n / 2}^{2}+\sum_{m=1}^{(n-2) / 2}\left(x_{m}^{2}+y_{m}^{2}\right)=\frac{4}{2 n-3},
$$

respectively.

We summarize these results in the following theorem and corollaries.

THEOREM 1. Let the function

$$
P(z)=1+p_{1} z+\cdots+p_{n} z^{n}+\cdots
$$

be regular and have a positive real part in $|z|<1$. Then $\left|p_{n}\right| \leqq 2$, and $p_{n}=2$ for $a$ given $n$ when, and only when, $P(z)$ is of the form

$$
P(z)=\frac{1+p_{1} z+p_{2} z^{2}+\cdots+p_{n-1} z^{n-1}+z^{n}}{1-z^{n}}, \quad p_{n-k}=p_{k}, 0<k<n,
$$

and the coefficient space $E_{n-1}$ of $P(z)$ is the closed $(n-1)$-simplex determined by the equations (3.17). The vertices of the $(n-1)$-simplex are given by (3.23) and (3.24) and the boundary hyperplanes by the equations (3.25) and (3.26).

COROLlary 1. If $n$ is an odd positive integer $>1$ and

$$
\left|p_{1}\right|^{2}+\cdots+\left|p_{(n-1) / 2}\right|^{2} \leqq \frac{2}{n-1},
$$

or if $n$ is an even integer $\geqq 2$ and 


$$
\left|p_{1}\right|^{2}+\cdots+\left|p_{n / 2}\right|^{2} \leqq \frac{4}{2 n-3}
$$

then the function

$$
P(z)=\left(1+p_{1} z+p_{2} z^{2}+\cdots+p_{n-1} z^{n-1}+z^{n}\right) \div\left(1-z^{n}\right),
$$

$p_{n-k}=p_{k}, 2 k \leqq n$, has $\operatorname{Re} P(z)>0$ in $|z|<1$.

COROLlary 2. With the notation of Theorem 1 the boundary of the $(n-1)$ simplex $E_{n-1}$ given by (3.17) is determined from the equation $\Delta_{n}=0, n>1$, where $\Delta_{n}$ is the determinant

$$
\Delta_{n}=\left|\begin{array}{lllll}
2 & p_{1} & p_{2} & \cdots & p_{n-1} \\
p_{1} & 2 & p_{1} & \cdots & p_{n-2} \\
p_{2} & p_{1} & 2 & \cdots & p_{n-3} \\
\vdots & \vdots & \vdots & \vdots \\
\bar{p}_{n-1} & \dot{p}_{n-2} & \dot{p}_{n-3} & \cdots & \dot{2}
\end{array}\right|=2^{n}(n !)^{2} \sin ^{2} \frac{\pi}{n} \cdot \prod_{k=1}^{n} \lambda_{k}
$$

Proof of Corollary 2. Using the representation (3.17) for the coefficients $p$, we write $\Delta_{n}$ as the product of two determinants, $\Delta_{n}=2^{n} A_{n} B_{n}$, where

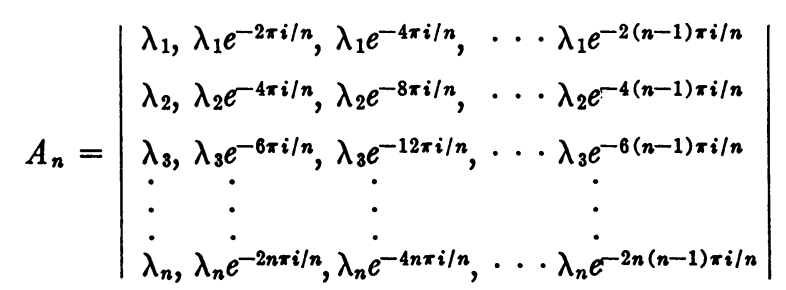

and $B_{n}$ is the determinant $A_{n}$ with the $\lambda$ 's replaced by 1 's and $i$ replaced by $-i$. Thus $\prod_{1}^{n} \lambda_{k}$ is a factor of $A_{n}$ and we obtain

$$
\Delta_{n}=2^{n}\left(\prod_{1}^{n} \lambda_{k}\right)\left|B_{n}\right|^{2} \geqq 0 .
$$

In the determinant $B_{n}$ except for the first column, the elements in the columns add up to zero, so that the order of $B_{n}$ is easily reduced a step at a time. Thus

$$
\begin{aligned}
\Delta_{n} & =2^{n}\left(\prod_{1}^{n} \lambda_{k}\right) n^{2}(n-1)^{2} \cdots 3^{2}\left\|\begin{array}{cc}
1 & 1 \\
1 & e^{2 \pi i / n}
\end{array}\right\|^{2} \\
& =2^{n}(n !)^{2} \sin ^{2} \frac{\pi}{n}\left(\prod_{1}^{n} \lambda_{k}\right) \\
& >0
\end{aligned}
$$

Since a boundary point of $E_{n-1}$ corresponds to a $\lambda_{k}$ having the values 0 or 1 , 
$\Delta_{n}=0$ defines the boundary of $E_{n-1}$ for $n>1$. When $n=1, \Delta_{1}=2$ and $E_{0}$ is the point $p_{1}=2$.

Although we have confined our attention to extremal functions for which $\operatorname{Re} p_{n}$ is a maximum, the formula (2.8) for $\delta p_{n}$ may be used to determine the extremal functions $P(z)$ which maximize $\operatorname{Re} F\left(p_{1}, p_{2}, \cdots, p_{n}\right)$ where $F\left(p_{1}, \cdots, p_{n}\right)$ is any continuous function having continuous partial derivatives in an open set containing the coefficient space $V_{n}\left(p_{1}, \cdots, p_{n}\right)$ of the class $\rho$ and for which the partial derivatives $\lambda_{k}=\partial F / \partial p_{k}, k=1, \cdots, n$, are not all zero at the point $\left(p_{1}, \cdots, p_{n}\right)$ determined by the extremal function. We find that the extremal function maximizes $\operatorname{Re}\left(\sum_{1}^{n} \lambda_{k} p_{k}\right)$ and is the function

$$
P(z)=\frac{\sum_{k=1}^{n} k\left(\lambda_{k} \sum_{s=0}^{k} \frac{p_{s}}{z^{k-s}}+\bar{\lambda}_{k} \sum_{s=0}^{k} \bar{p}_{s} z^{k-s}\right)}{\sum_{k=1}^{n} k\left(\frac{\lambda_{k}}{z^{k}}-\bar{\lambda}_{k} z^{k}\right)}, \quad p_{0}=1 .
$$

It is seen from (3.29) that the extremal functions $P(z)$ maximizing $\operatorname{Re} F$ have the property that the real part of $P(z)$ vanishes identically on $|z|=1$.

In particular, one may obtain the extremal functions which minimize the Toeplitz form

$$
F=\sum p_{\mu-\nu} X_{\nu} \bar{X}_{\mu}
$$

$F$ then turns out to be non-negative in accordance with the CarathéodoryToeplitz theory. We omit the details.

Turning to another problem we shall now see how the variational technique easily leads to the well-known inequality

$$
\operatorname{Re} P(z) \geqq \frac{1-r}{1+r}, \quad|z|=r<1,
$$

for functions of class $P$.

Let $P_{0}(z)$ be an extremal function for which, when $z$ is fixed in the unit circle, $\operatorname{Re} P_{0}(z)$ is a minimum for the class $\beta$. By a rotation in the $z$-plane we may assume $z$ to be a positive number $r$. Since $\operatorname{Re} \delta P_{0}(r) \geqq 0$ in $(2.7)$ we have $\operatorname{Re} e^{i \theta} A \leqq 0$ for all $\theta$ where

$$
\begin{aligned}
A=A(r)= & \left(\frac{z_{0} P_{0}^{\prime}(r)}{P_{0}\left(z_{0}\right)}-1\right) \frac{r}{z_{0}\left(z_{0}-r\right)}+\left(\frac{z_{0} P_{0}(r)}{P_{0}\left(z_{0}\right)}-r\right) \frac{r}{z_{0}\left(z_{0}-r\right)^{2}} \\
& +\frac{\left(P_{0}^{\prime}(r)\right)^{-}}{P_{0}\left(z_{0}\right)} \cdot \frac{r^{2}}{1-z_{0} r}+\left(\frac{\left(P_{0}(r)\right)^{-}}{P_{0}\left(z_{0}\right)}+1\right) \frac{r}{\left(1-z_{0} r\right)^{2}} .
\end{aligned}
$$

Since $\theta$ is arbitrary it follows that $A=0$. Replacing $z_{0}$ by $z$ and solving the equation $A=0$ for $P_{0}(z)$ we obtain 


$$
\begin{aligned}
& \left(1-r^{2}\right)\left(1-z^{2}\right) P_{0}(z)=A_{3} z^{3}+A_{2} z^{2}+A_{1} z+A_{0}, \\
& A_{0}=-r P_{0}^{\prime}(r)+r^{2}\left(P_{0}^{\prime}(r)\right)^{-}+P_{0}(r)+r^{2}\left(P_{0}(r)\right)^{-}=\left(1-r^{2}\right) P_{0}(0) \\
& =1-r^{2} \text {, } \\
& A_{1}=\left(1+2 r^{2}\right) P_{0}^{\prime}(r)-\left(2 r^{2}+r^{4}\right)\left(P_{0}^{\prime}(r)\right)^{-}-2 r\left\{P_{0}(r)+\left(P_{0}(r)\right)^{-}\right\} \text {, } \\
& A_{2}=\left(2 r^{3}+r\right)\left(P_{0}^{\prime}(r)\right)^{-}-\left(2 r+r^{3}\right) P_{0}^{\prime}(r)+r^{2} P_{0}(r)+\left(P_{0}(r)\right)^{-} \text {, } \\
& A_{8}=r^{2}\left\{P^{\prime}(r)-\left(P_{0}^{\prime}(r)\right)-\right\} \text {. }
\end{aligned}
$$

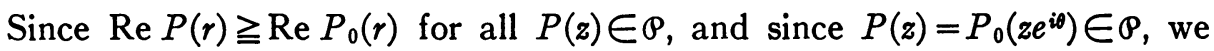
have $\operatorname{Re} P_{0}\left(r e^{i \theta}\right) \geqq \operatorname{Re} P_{0}(r)$ for all $\theta$. Thus $\operatorname{Re} P_{0}(r)$ is a minimum value of $\operatorname{Re} P_{0}\left(r e^{i \theta}\right)$ as a function of $\theta$. It follows from the Cauchy-Riemann equations that

$$
I P_{0}^{\prime}(r)=\left.\frac{\partial}{\partial r} I P_{0}\left(r e^{i \theta}\right)\right|_{\theta=0}=-\left.\frac{1}{r} \frac{\partial}{\partial \theta} \operatorname{Re} P_{0}\left(r e^{i \theta}\right)\right|_{\theta=0}=0 .
$$

Thus $P_{0}^{\prime}(r)$ is real. Then $A_{3}=0$. Since $A_{0}$ is real it follows that $\left\{P_{0}(r)+r^{2}\left(P_{0}(r)\right)^{-}\right\}$and $\left\{P_{0}(r)+\left(P_{0}(r)\right)^{-}\right\}$are both real. This implies that $P_{0}(r)$ is real. Since $A_{2}-A_{0}=\left(1-r^{2}\right)\left\{\left(P_{0}(r)\right)^{-}-P_{0}(r)\right\}$, we have $A_{2}=A_{0}$ $=1-r^{2}$. Also $A_{1}$ is seen to be real. We have now seen that $P_{0}(z)$ is of the form

$$
P_{0}(z)=\frac{1+k z+z^{2}}{1-z^{2}}
$$

$k$ real.

Since $P_{0}(r) \geqq 0$, we have $k \geqq-2$. Since $P_{0}(r)$ is minimal for the class $P$ we must take $k=-2$. In this case

$$
P_{0}(z)=\frac{1-z}{1+z}
$$

so that (3.30) follows with equality holding only for the function $P_{0}(\epsilon z)$, $|\epsilon|=1$.

It should be noticed that the equation $A_{0}=1-r^{2}$ may not be treated as a differential equation for finding $P_{0}(r)$ unless it is first shown that the extremal function $P_{0}$ does not vary with $r$.

4. Interior variations for subordinate functions. Let the analytic function

$$
f(z)=A_{1} z+A_{2} z^{2}+\cdots+A_{n} z^{n}+\cdots, \quad f(0)=0, A_{1} \neq 0,
$$

be regular and univalent in $|z|<1$. Let

$$
F(z)=a_{1} z+a_{2} z^{2}+\cdots+a_{n} z^{n}+\cdots, \quad F(0)=0,
$$

be regular and subordinate to $f(z)$ in $|z|<1$. Then

$$
F(z)=f(\omega(z))
$$

where $\omega(z)$ is regular in $|z|<1, \omega(0)=0,|\omega(z)|<1$ for $|z|<1$. We may write $\omega(z)$ in the form 


$$
\omega(z)=\frac{P(z)-1}{P(z)+1}
$$

where $P(z)$ is a member of class $P$. We also denote by $z=\phi(w)$ the inverse function of $w=f(z)$. If $P^{*}(z)$ is given by (2.6) upon varying $P(z)$, and if $\omega^{*}(z)$ corresponds by (4.4) to $P^{*}(z)$, we easily obtain

$$
\begin{aligned}
\omega^{*}(z) & =\frac{P^{*}(z)-1}{P^{*}(z)+1}=\frac{P(z)-1}{P(z)+1}+\rho^{2} \lambda(z)+o\left(\rho^{2}\right) \\
& =\omega(z)+\rho^{2} \lambda(z)+o\left(\rho^{2}\right)
\end{aligned}
$$

where

$$
\lambda(z)=-\frac{1}{2}\left(1-\left|z_{0}\right|^{2}\right)(1-\phi(F))^{2} A(z)
$$

and where $A(z)$ is defined as the right-hand side of equation (2.7) omitting the term $o(1)$. We also have

$$
\begin{aligned}
P(z) & =\frac{1+\phi(F)}{1-\phi(F)}, \quad(P(z)+1)^{2}=4(1-\phi(F))^{-2}, \\
P^{\prime}(z) & =\frac{2 \phi^{\prime}(F) F^{\prime}(z)}{(1-\phi(F))^{2}} .
\end{aligned}
$$

We now write $A(z)$ in the form

$$
\begin{aligned}
A(z)= & \frac{2 \phi^{\prime}(F) F^{\prime}(z)}{(1-\phi(F))^{2}}\left\{\frac{e^{i \theta} z}{P\left(z_{0}\right)\left(z_{0}-z\right)}+\frac{z^{2} e^{-i \theta}}{\left(P\left(z_{0}\right)\right)^{-\left(1-\bar{z}_{0} z\right)}}\right\} \\
& +\frac{1+\phi(F)}{1-\phi(F)}\left\{\frac{e^{i \theta} z}{P\left(z_{0}\right)\left(z_{0}-z\right)^{2}}+\frac{e^{-i \theta} z}{\left(P\left(z_{0}\right)\right)^{-}\left(1-\bar{z}_{0} z\right)^{2}}\right\} \\
& +\left\{\frac{e^{-i \theta} z}{\left(1-\bar{z}_{0} z\right)^{2}}-\frac{e^{i \theta} z^{2}}{z_{0}\left(z_{0}-z\right)^{2}}-\frac{e^{i \theta} z}{z_{0}\left(z_{0}-z\right)}\right\}
\end{aligned}
$$

where

$$
\begin{aligned}
P\left(z_{0}\right) & =\frac{1+\phi\left(F\left(z_{0}\right)\right)}{1-\phi\left(F\left(z_{0}\right)\right)} \\
F^{*}(z) & =f\left(\omega(z)+\rho^{2} \lambda(z)+o\left(\rho^{2}\right)\right) \\
& =F(z)+f^{\prime}(\omega(z)) \lambda(z) \rho^{2}+o\left(\rho^{2}\right) \\
& =F(z)+\frac{\lambda(z)}{\phi^{\prime}(F)} \rho^{2}+o\left(\rho^{2}\right) .
\end{aligned}
$$


Thus the variational formula for analytic functions $F(z)$ subordinate to a given univalent function $f(z)$ in $|z|<1$ is given by

$$
F^{*}(z)=F(z)-\rho^{2}\left(1-\left|z_{0}\right|^{2}\right) \frac{(1-\phi(F))^{2}}{2 \phi^{\prime}(F)} A(z)+o\left(\rho^{2}\right)
$$

where $A(z)$ is defined by (4.9) and $\phi$ is the inverse of $f$.

\section{REFERENCES}

1. C. Carathéodory, Über den Variabilitätsbereich der Fourierschen Konstanten von positiven harmonischen Funktionen, Rend. Circ. Mat. Palermo 32 (1911), 193-217.

2. B. Detwiler and W. C. Royster, A variational formula for functions convex in the direction of the imaginary axis, Abstract 568-2, Notices Amer. Math. Soc. 7 (1960), 242.

3. E. Fischer, Über das Carath\&odorysche Problem, Potenzreihen mit positivem reellen Teil betreffend, Rend. Circ. Mat. Palermo 32 (1911), 240-256.

4. U. Grenander and G. Szegö, Toeplitz forms and their applications, Univ. of California Press, Berkeley, 1958.

5. J. A. Hummel, $A$ variational method for star-like functions, Proc. Amer. Math. Soc. 9 (1958), 82-87.

6. W. Kaplan, Close-to-convex schlicht functions, Michigan Math. J. 1 (1952), 169-185.

7. F. Riesz, Über ein Problem des Herrn Carathéodory, J. Reine Angew. Math. 146 (1915), 83-87.

8. M. M. Schiffer, Applications of variational methods in the theory of conformal mapping, Proc. Symp. Appl. Math. Vol. 8, McGraw-Hill, New York, 1958.

9. O. Toeplitz, Über die Fouriersche Entwicklung positiver Funktionen, Rend. Circ. Mat. Palermo 32 (1911), 191-192.

10. M. Tsuji, On a regular function, whose real part is positive in a unit circle, Proc. Japan Acad. 21 (1945), 321-329.

Rutgers, The State University, New Brunswick, New Jersey 